\title{
Stability of Planar Switched Systems under Delayed Event Detection
}

\author{
Benoît Legat ${ }^{1}$, Cláudio Gomes ${ }^{2}$, Paschalis Karalis ${ }^{3}$, \\ Raphaël M. Jungers ${ }^{4}$, Eva M. Navarro-López ${ }^{5}$, and Hans Vangheluwe ${ }^{6}$
}

\begin{abstract}
In this paper, we analyse the impact of delayed event detection on the stability of a 2-mode planar hybrid automata. We consider hybrid automata with a unique equilibrium point for all the modes, and we find the maximum delay that preserves stability of that equilibrium point. We also show for the class of hybrid automata treated that the instability of the equilibrium point for the equivalent hybrid automaton with delay in the transitions is equivalent to the existence of a closed orbit in the hybrid state space, a result that is inspired by the Joint Spectral Radius theorem. This leads to an algorithm for computing the maximum stable delay exactly. Other potential applications of our technique include co-simulation, networked control systems and delayed controlled switching with a state feedback control.
\end{abstract}

\section{INTRODUCTION}

In order to make the computation of the behaviour of hybrid systems possible, one needs to not only approximate the continuous dynamics, but also to accurately and efficiently detect when to compute the discrete-event dynamics. In order to accurately do so, transition (or event) detection and location schemes are employed. The efficiency requirement is satisfied by setting the appropriate parameters of the transition location scheme. Wrong tolerance values can lead to unnecessary computations and/or inaccurate results (see, e.g., Section II).

Contributions. In this paper, we focus on the numerical stability of hybrid system simulation. We formalize the problem of finding the Maximum Stable Delay (MSD), that is, the maximum delay a hybrid system can admit in a transition, so that its solutions remain asymptotically stable (Section III).

Inspired by the Joint Spectral Radius theorem [1], we show how this problem can be solved for a restricted class of planar hybrid systems, by reducing it to a problem of finding a non-trivial closed orbit in the transition-delayed system Section IV. As an example application of these theoretical results, we provide an algorithm to find such a closed orbit in a non-linear hybrid system (Section V].

${ }^{1}$ B. L. is a F.R.S.-FNRS Research Fellow, at UCLouvain.

${ }^{2}$ C. G. is a FWO Research Fellow, at the University of Antwerp, supported by the Research Foundation - Flanders (File Number 1S06316N).

${ }^{3} \mathrm{P}$. K. is a research engineer at Cadence Design Systems.

${ }^{4}$ R. M. Jungers is a F.R.S.-FNRS honorary Research Associate, at UCLouvain. He is supported by the Walloon Region and the Innoviris Foundation.

${ }^{5}$ E. M. N. is a Honorary Senior Research Fellow at the University of Manchester.

${ }^{6} \mathrm{H}$. V. is a Professor at the University of Antwerp. His work is partially supported by Flanders Make vzw, the strategic research centre for the manufacturing industry.
The MSD can then be used to set the appropriate parameterization of transition detection and location schemes, and/or the size of the simulation step. Other potential applications include: (i) networked control systems (see, e.g., [2]), where the component responsible for deciding the mode of the plant may be reacting to a delayed signal; and (ii) real-time simulation of hybrid systems, where state transition location can be disabled, or relaxed, to increase performance, as in animation of colliding multi-body systems [3].

An extended version of this work is available in [4, Chapter 6].

Related Work. In the domain of network control, [5] focuses on the state-feedback stabilization of LTI systems that can be remotely controlled over a multi-hop network. In contrast, we focus on non-linear planar systems with a switching surface. We follow an approach that is similar to [6], [2], [7]. Prior work [6] explores how to use Lyapunov functions to approximate the MSD in the particular case of affine systems, without resorting to finding closed orbits. However, no formal proof is provided regarding the correctness of the procedure. In [2], the delayed system is formulated as a hybrid system. Then, the stability of which is proven if a Lyapunov function can be found, using the sum of squares [8] approach, which can only approximate the MSD. The work in [7] provides a comprehensive study of the dynamics of planar systems with the form $\dot{x}=$ $A x+\operatorname{sign}\left(w^{T} x\right) v$, with $A$ a constant matrix. Similarly to the current manuscript, Poincar maps are used to analyze the stability of periodic motions ([7, Section 5.2]). More recently, [9] studied the maximum sampling time $\tau$ such that the asymptotic stability is preserved under the discretization of a hybrid nonlinear system given that the length of the (not necessarily equal) sampling intervals does not exceed $\tau$. While the problem setting is similar to our work, their aim is to prove the existence of a maximal sampling time $\tau$, not to approximate it. We refer the reader to [10] for similar work restricted to linear.

\section{Motivating Example: Relaxed Bouncing Ball}

Consider a bouncing ball modeled with two modes: freefall, and contact. The detailed equations for each mode are given in [4, Section 6.2].

The ball changes from free fall mode to contact mode when it comes in contact with the floor. Formally, that is when $g(x)=\left[\begin{array}{ll}1 & 0\end{array}\right] x-r \leq 0$, with $r$ denoting the radius of the ball. 
[4, Figure 6.1] shows an example trajectory, which converges to an equilibrium in the contact mode. In a simulation, the moment that the ball changes from the free-fall mode to the contact mode is approximated using a state transition location technique. [4, Figure 6.2] shows a simulation where the transition from free-fall to contact mode is not correctly detected, resulting in a delay of $0.002 \mathrm{~s}$. The simulation shows the ball reaching the same compression that it had on its initial state. Since the total energy of the physical bouncing ball is dissipated via air friction and impact damping, the bouncing ball simulation should come to a rest. Instead, [4, Figure 6.2] shows that the simulation of the bouncing ball with transition delays of up to $0.002 \mathrm{~s}$ may fail to come to a rest.

The research question follows: for a given hybrid system whose trajectories eventually tend to an equilibrium, what is the largest delay in the transition the simulations can tolerate, such that that property is preserved? The next section formulates this problem.

\section{PROBLEM Formulation}

We adopt the usual definition of Hybrid Automaton and the notation in [4, Definition 26].

Definition 1 (Hybrid automaton [11]): A hybrid automaton $\mathcal{S}$ is a collection

$$
\mathcal{S}=(Q, E, \mathcal{X}, \text { Dom }, \mathcal{F}, \text { Guard, } R)
$$

where: $\bullet Q$ is a finite set of modes; $\bullet E \subseteq Q \times Q$ is a finite set of edges called transitions; $\bullet \mathcal{X} \subseteq \mathbb{R}^{n}$ is the continuous state space, for some natural $n$; $\bullet$ Dom : $Q \rightarrow 2^{\mathcal{X}}$ is the mode domain; $\bullet \mathcal{F}=\left\{f_{q}(x): q \in Q\right\}$ is a collection of time-invariant vector fields such that each $f_{q}(x)$ is Lipschitz continuous on $\operatorname{Dom}(q)$; $\bullet$ Guard $: E \rightarrow 2^{\mathcal{X}}$ defines a guard set for each transition. $\bullet R: E \times \mathcal{X} \rightarrow 2^{\mathcal{X}}$ specifies how the continuous state is reset at each transition.

The Lipschitz continuity assumption in Definition 1 ensures the existence and continuity of a solution inside a mode as shown by [4, Lemma 1].

Definition 2 (Flow): Given a dynamical system $\dot{x}(t)=$ $f(x(t))$, the flow of the vector field $f$, denoted $\phi_{f}(x, t)$, is the state of the system after a time $t$ if the initial state is $x$.

Example 1: Fig. 1 shows the graphical representation of the hybrid automaton for the bouncing ball described in Section II

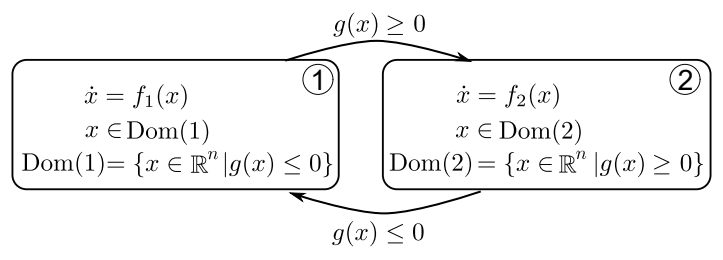

Fig. 1: A Bi-modal Hybrid Automata in the notation used by [12].

Definition 3 (Hybrid trajectory. [13, Definition 2.37): A hybrid trajectory of a system $\mathcal{S}$ as defined in Definition 1 is a subset $\mathcal{T} \subseteq Q \times \mathbb{R} \times \mathcal{X}$ with

$\mathcal{T}=\bigcup_{j=0}^{J-1}\left\{q_{j}\right\} \times\left\{(t, x) \mid t_{j} \leq t \leq t_{j+1}, x=\phi_{f_{q_{j}}}\left(x_{j}, t-t_{j}\right) \in \operatorname{Dom}\left(q_{j}\right)\right\}$

for some finite sequence of times $t_{0}<t_{1}<\cdots<t_{J}$, modes $q_{j} \in Q$ and state vector $x_{j} \in \operatorname{Dom}\left(q_{j}\right)$ such that $\left(q_{j-1}, q_{j}\right) \in$ $E, y_{j} \in \operatorname{Guard}\left(\left(q_{j-1}, q_{j}\right)\right)$ and $x_{j} \in R\left(\left(q_{j-1}, q_{j}\right), y_{j}\right)$ for $j=1, \ldots, J$ where $y_{j}=\phi_{f_{q_{j-1}}}\left(x_{j-1}, t_{j}-t_{j-1}\right)$.

Note that Definition 3 excludes chattering and Zeno behaviour, which is assumed inexistent later by assumption 2 but considered in [13, Definition 2.3]. The later assumption also allows restricts the non-determinism of hybrid automata while still permitting non trivial behaviour.

We define the following notations for trajectories:

$$
\begin{aligned}
\mathcal{T}[\underline{t}, \bar{t}] & =\{(q, t, x) \in \mathcal{T} \mid \underline{t} \leq t \leq \bar{t}\}, \\
\left.\mathcal{T}\right|_{\mathcal{X}} & =\{x \mid \exists q, t:(q, t, x) \in \mathcal{T}\} \\
t_{\min }(\mathcal{T}) & =\inf _{(q, t, x) \in \mathcal{T}} t \quad t_{\max }(\mathcal{T})=\sup _{(q, t, x) \in \mathcal{T}} t \\
\mathcal{T}_{1} \uplus \mathcal{T}_{2} & =\mathcal{T}_{1} \cup\left\{\left(q, t+t_{\max }\left(\mathcal{T}_{1}\right)-t_{\min }\left(\mathcal{T}_{2}\right), x\right) \mid(q, t, x) \in \mathcal{T}_{2}\right\} .
\end{aligned}
$$

Note that if $\mathcal{T}$ is a hybrid trajectory then so is $\mathcal{T}[\underline{t}, \bar{t}]$, which allows us to define the combined notation $\mathcal{T}[\underline{t}, \bar{t}] \mid \mathcal{X}$. However, even if $\mathcal{T}_{1}$ and $\mathcal{T}_{2}$ are hybrid trajectories, $\mathcal{T}_{1} \uplus \mathcal{T}_{2}$ may not be a hybrid trajectory if for instance it induces a transition that does not respect a guard set.

We say that an equilibrium of a hybrid automaton is Globally asymptotically stable $(G A S)$ if all trajectories of the system remain close, and converge, to the equilibrium (see, e.g., [13]).

As motivated in Section II we need to analyze the behavior of a hybrid automaton under delay in the transition. It turns out that we can construct a hybrid automaton for which the stability is equivalent to the stability under delay in the transition of the original system. We present this reduction in the following restricted class of hybrid automata depicted in Fig. 1.

Definition 4 (Bi-modal Hybrid Automata): A Bi-modal Hybrid Automaton (BMHA) system is a hybrid automaton as defined in Definition 1 such that there exists a continuously differentiable function $g$ such that $Q=\{1,2\}$, $\operatorname{Dom}(1)=\{x \mid g(x) \leq 0\}, \operatorname{Dom}(2)=\{x \mid g(x) \geq 0\}$, $E=\{(1,2),(2,1)\}, \operatorname{Guard}((1,2))=\{x \mid g(x) \geq 0\}$, $\operatorname{Guard}((2,1))=\{x \mid g(x) \leq 0\}$ and $R((1,2), x)=$ $R((2,1), x)=\{x\}$ for all $x \in \mathbb{R}^{n}$. This is illustrated in Fig. 1

Definition 5: Given a BMHA $\mathcal{S}$, as defined in Definition 4, and a delay $H>0$, we define its transition-delayed $\mathcal{S}_{H}$ counterpart as in [4, Figure 6.4].

$\mathcal{S}_{H}$ is non-deterministic, having trajectories where a transition can be delayed by a maximum of $H$ units of time. Moreover, $\mathcal{S}_{H}$ has the same equilibrium point as the original BMHA system. And the equilibrium point of $\mathcal{S}_{H}$ may not be GAS, even though the original system $\mathcal{S}$ is GAS, as exemplified in [4, Figure 6.2].

The following results follow from Definitions 4 and 5 and lead to our problem formulation. 
Proposition 1: If $\mathcal{T}$ is a trajectory of $\mathcal{S}_{H^{\prime}}$, then it is also a trajectory of $\mathcal{S}_{H}$, for any $H^{\prime} \leq H$.

Corollary 1: For any $H^{\prime} \leq H$, if the equilibrium $x^{*} \in \mathbb{R}^{n}$ of $\mathcal{S}_{H}$ is GAS, then the equilibrium $x^{*}$ of $\mathcal{S}_{H^{\prime}}$ is also GAS.

The previous results naturally prompt the following problem.

Problem 1: Given a BMHA $\mathcal{S}$, as defined in Definition 4 with a GAS equilibrium $x^{*}$, find

$$
\sigma(\mathcal{S})=\sup _{H \geq 0} H \text { s.t. } x^{*} \text { is a GAS equilibrium of } \mathcal{S}_{H}
$$

We denote the switching surface as

$$
G=\left\{x \in \mathbb{R}^{2} \mid g(x)=0\right\} .
$$

To avoid pathological cases, we assume that the single equilibrium is not in the switching surface. Furthermore, without loss of generality, we consider that the equilibrium is in the interior of $\operatorname{Dom}(1)$ and assume that it is GAS for the mode 1 .

We call $\sigma(\mathcal{S})$ the Maximum Stable Delay (MSD). Intuitively, $\sigma(\mathcal{S})$ gives us the maximal delay for which the GAS property $x^{*}$ is preserved under delayed switching: by Corollary 1, $x^{*}$ of $\mathcal{S}_{H}$ is GAS for all $H<\sigma(\mathcal{S})$. Knowing $\sigma(\mathcal{S})$ allows one to define the time step of a simulation algorithm, or the tolerance of a state transition location scheme.

Problem 1 can be extended to a general hybrid automaton (Definition 1) because the construction of $\mathcal{S}_{H}$ can be generalized. We focus on BMHA because our subsequent results cannot be straightforwardly generalized. This is left as future work.

Problem 1 can be reduced to the stability of an equilibrium of a hybrid automaton of the form given in [4, Figure 6.4], and thus we can leverage classical tools developed for the stability of hybrid automata. For example, [4, Section 6.5.1] demonstrates the use of SpaceEx to prove the stability of $\mathcal{S}_{H}$, for a given $H$. However, deciding the stability of an equilibrium of a hybrid automaton is not possible in general [14], hence this does not provide an efficient approach and it only approximates the $\sigma(\mathcal{S})$ of problem 1 .

As we show in the following section, under mild assumptions, the stability of the subclass of planar hybrid automata of the form given in [4, Figure 6.4] is equivalent to the existence of a closed orbit (Definition 6). As shown in Section V, this theoretical result leads to practical algorithms to compute $\sigma(\mathcal{S})$ with arbitrary accuracy.

\section{ORBIT AND STABILITY}

Our approach is similar to the stabilization of a constrained linear switched system [15], [16]. In the theory of constrained switched systems, the asymptotic stability, can be proven by showing that there are no closed orbits in the system (see [15, Theorem A], [17], and example applications in [18]). We show that there is an equivalence between the absence of cyclic behaviors in the transition-delayed counterpart Definition 5, and its asymptotic stability. We start by defining the notion of closed orbit, to formalize cyclic trajectories.
Definition 6 (Closed orbit): Given a hybrid trajectory $\mathcal{T}$ as given in Definition 3, we say that it is a closed orbit if there exists

$$
\left(q_{0}, t_{0}, x_{0}\right),(\tilde{q}, \tilde{t}, \tilde{x}),(\bar{q}, \bar{t}, \bar{x}) \in \mathcal{T}
$$

such that $\bar{q}=q_{0}, t_{0}<\tilde{t}<\bar{t}$ and $\tilde{x} \neq \bar{x}=x_{0}$.

The condition $\tilde{x} \neq x$ excludes the equilibrium from being a trivial closed orbit.

Proposition 2: If there is a closed orbit in $\mathcal{S}_{H}$, then the equilibrium $x^{*}$ of $\mathcal{S}_{H}$ is not GAS.

The above proposition allows, for a given $H$, to prove the instability of $x^{*}$ of $\mathcal{S}_{H}$ by just finding a closed orbit. By trying to find the smallest $H$ for which there is a closed orbit, one can approximate from above the solution $\sigma(\mathcal{S})$ to problem 1. However, approximation from above may not be sufficient. To see why, imagine that the smallest $H$ for which there exists a closed orbit in $\mathcal{S}_{H}$ is found. Then, it might still be the case that there exists a $H^{\prime}<H$ such that $x^{*}$ in $\mathcal{S}_{H^{\prime}}$ is not GAS. Our contribution is to show that such $H^{\prime}$ cannot exist for a class of planar BMHA systems satisfying assumptions 1 to 4 , formalized below.

We use classical results of analysis of planar non-linear systems such as continuity and monotonicity of a Poincaré Map. Such classical notions need to be carefully used in this setting since they are usually developed for non-hybrid systems. We start by discussing the monotonicity. We will see in Corollary 2 that the Poincare Map is monotonous for the hybrid automaton $\mathcal{S}$. However, for a non-deterministic hybrid automaton such as $\mathcal{S}_{H}$, monotonicity of the Poincaré Map is not guaranteed as two trajectories can intersect as long as they are in different modes. Nevertheless, the topological argument commonly used to prove monotonicity (see e.g. [19, Section 10.4]) can still be used to obtain the result given in Lemma 1 for planar BMHA systems.

We start by defining the following connectedness concepts from topology; see e.g. [20, Chapter 3].

Definition 7 (Path): Given a set $X$ and two points $x, y \in$ $X$, a path in $X$ from $x$ to $y$ is the image of a continuous map $f:[0,1] \rightarrow X$ such that $f(0)=x$ and $f(1)=y$. We denote the union of all paths in $X$ from $x$ to $y$ as $[x, y]_{X}$.

Definition 8 (Path components of $X$ ): We define an equivalence relation on the set $X$ by defining $x \sim y$ if there is a path in $X$ from $x$ to $y$. The equivalence classes are called the path components of $X$.

Given a set $U$, we denote its closure by $\bar{U}$ and its interior by $\operatorname{int}(U)$.

Given the vector field $f_{1}$ of the first mode of a planar BMHA $\mathcal{S}$ defined in Definition 4, we assume the existence of a continuously differentiable function $s: \mathbb{R}^{2} \rightarrow \mathbb{R}$ such that the curve

$$
S_{p}=\left\{x \mid s(x)=0, \nabla s(x) \cdot f_{1}(x)>0\right\}
$$

satisfies $S_{p} \cap G=\emptyset$, where $G$ is the switching surface. We refer to the closed section $S_{p}$ as Poincaré curve, its definition is similar to the notion of local sections; see [19, Section 10.2]. 
Assumption 1: The Poincaré curve $S_{p}$ has a single path component, $\overline{S_{p}}=S_{p} \cup\left\{x^{*}\right\}$ and the restriction of the Euclidean norm to $S_{p}$ is injective.

We use the following notations for the return time and Poincaré Map, given a vector field $f$ and a set $U$ :

$$
\begin{aligned}
& \tau_{U}^{f}(x)=\inf \left\{t \mid t>0, \phi_{f}(x, t) \in U\right\}, \\
& P_{U}^{f}(x)=\phi_{f}\left(x, \tau_{U}^{f}(x)\right) .
\end{aligned}
$$

Note that $P_{U}^{f}(x)$ is not defined when $\tau_{U}^{f}(x)$ is infinite.

Given a trajectory $\mathcal{T}$, a set $U$ and a time $t_{0} \in \mathbb{R}$, we define the following notation

$$
\begin{aligned}
\tau_{U}^{\mathcal{T}}\left(t_{0}\right) & =\inf \left\{t \mid(q, t, x) \in \mathcal{T}, t>t_{0}, x \in U\right\} \\
\mathcal{P}_{U}^{\mathcal{T}}\left(t_{0}\right) & =\left\{(q, t, x) \in \mathcal{T} \mid t=\tau_{U}^{\mathcal{T}}\left(t_{0}\right)\right\} .
\end{aligned}
$$

The following lemma shows that, whenever a trajectory $\mathcal{T}$ of $\mathcal{S}_{H}$ intersects with itself, it must be under a different mode. [4, Figure 6.5] gives representative examples of this situation.

Lemma 1: Given a planar hybrid automaton $\mathcal{S}_{H}$ as given in Definition 5, with a Poincaré curve $S_{p}$ as given in (2) and a trajectory $\mathcal{T}$ of $\mathcal{S}_{H}$, consider $\left(q_{1}, t_{1}, x_{1}\right) \in \mathcal{T}$ with $x_{1} \in S_{p}$ and $\left(q_{2}, t_{2}, x_{2}\right) \in \mathcal{P}_{S_{p}}^{\mathcal{T}}\left(t_{1}\right)$ with $\left\|x_{2}-x^{*}\right\|_{2} \geq\left\|x_{1}-x^{*}\right\|_{2}$. Under assumption 1, there exists a path component $U$ of $\mathcal{X} \backslash\left(\mathcal{T}\left[t_{1}, t_{2}\right] \mid \mathcal{X} \cup\left[x_{2}, x_{1}\right]_{S_{p}}\right)$ such that:

(1) $\left\{x \in S_{p} \mid\left\|x-x^{*}\right\|_{2} \leq\left\|x_{2}-x^{*}\right\|_{2}\right\} \subseteq \bar{U}$; and

(2) there exists $\epsilon>0$ such that $\mathcal{T}\left[t_{2}, t_{2}+\epsilon\right] \mid \mathcal{X} \cap U=\emptyset$.

Moreover, if $\left.\mathcal{T}\left[t_{2}, \infty\right]\right|_{\mathcal{X}} \cap U \neq \emptyset$ then the intersection $\left(q_{3}, t_{3}, x_{3}\right) \in \mathcal{P} \frac{\mathcal{T}}{U}\left(t_{2}\right)$ is such that there exists $\left(q_{4}, t_{4}, x_{3}\right) \in$ $\mathcal{T}\left[t_{1}, t_{2}\right]$ with $q_{3} \neq q_{4}$.

As a corollary, the monotonicity of the Poincaré Map holds for the mode 1, as stated in Corollary 2. However, when applying the same result to the delayed hybrid automaton, care must be taken to consider only trajectories that remain in mode 1 , as these are not affected by the delayed switching. If a trajectory of the delayed hybrid automaton remains in the domain of mode 1 between two consecutive intersections with the Poincaré Map, Corollary 2 shows that the second intersection has a smaller Euclidean norm. In fact, as shown by Lemma 2, this implies that the trajectory will remain in mode 1 until its end.

Corollary 2: Consider a planar hybrid automaton $\mathcal{S}_{H}$ as given in Definition 5 and a Poincaré curve $S_{p}$ as defined in 2). For all $x \in S_{p}$, if for any $t, \phi_{f}(x, t) \in \operatorname{Dom}(1)$, then $\left\|P_{S_{p}}^{f_{1}}(x)-x^{*}\right\|_{2}<\left\|x-x^{*}\right\|_{2}$.

Lemma 2: Given a delay $H$ and a planar hybrid automaton $\mathcal{S}_{H}$ as given in Definition 5, with a Poincaré curve $S_{p}$ as defined in (2), and a trajectory $\mathcal{T}$ of $\mathcal{S}_{H}$, consider $\left(1, t_{1}, x_{1}\right) \in \mathcal{T}$ with $x_{1} \in S_{p}$ and $\left(1, t_{2}, x_{2}\right) \in$ $\mathcal{P}_{S_{p}}^{\mathcal{T}}\left(t_{1}\right)$. Under assumption 1, if $\mathcal{T}\left[t_{1}, t_{2}\right] \mid \mathcal{X} \subseteq \operatorname{Dom}(1)$, then $\left.\mathcal{T}\left[t_{2}, \infty\right]\right|_{\mathcal{X}} \subseteq \operatorname{Dom}(1)$

In view of Corollary 2 and Lemma 2, if a trajectory does not intersect the switching surface between two intersections of the Poincaré curve (hence staying in mode 1) then it will repeat this behavior indefinitely. Therefore, as our aim is to find a closed orbit, we restrict our attention to points of the Poincare curve from which a trajectory of $\mathcal{S}$ intersects the switching surface. We denote the set of such points, illustrated in [4, Figure 6.6], as follows:

$$
\begin{aligned}
\tilde{S}_{p}=\left\{x \in S_{p} \mid \tau_{G}^{f_{1}}(x),\right. \text { is finite, } \\
\left.\qquad \nabla g\left(P_{G}^{f_{1}}(x)\right) \cdot f_{1}\left(P_{G}^{f_{1}}(x)\right)>0\right\} .
\end{aligned}
$$

Note that, by continuity of the functions $f_{1}$ and $g$, the value $g\left(P_{G}^{f_{1}}(x)\right) \cdot f_{1}\left(P_{G}^{f_{1}}(x)\right)$ in (3) cannot be negative if $x \in$ $\operatorname{Dom}(1)$, hence the condition $>0$ could be replaced by $\neq 0$. The condition simply excludes points $x$ such that $f_{1}\left(P_{G}^{f_{1}}(x)\right)$ is tangent to the switching surface.

Since the equilibrium $x^{*}$ is GAS for the mode 1 , by Corollary 2 and [4, Lemma 1], any trajectory of $\mathcal{S}_{H}$ (independently of $H$ ) starting at a point in $S_{p} \backslash \tilde{S}_{p}$ will converge to $x^{*}$. Lemma 3 provides a converse result, that is, if $x^{*}$ is not GAS and $\mathcal{S}_{H}$ does not admit any closed orbit, then subsequent intersections with $S_{p}$ will be farther from the origin. However, extra care must be taken for where to place the Poincaré curve, to make sure all its intersections with $S_{p}$ happen in mode 1 . Moreover, we need to assume that the dynamics of mode 2 , under the domain of mode 1 , do not prevent these intersections (e.g., it could lead to chattering [21, Section 1.2.4] around the switching surface, because any trajectory starting in mode 2 will cross the switching surface to mode 1 , by the stability of $x^{*}$ in $\mathcal{S}$ ).

The following assumption handles these scenarios. [4, Figure 6.7] illustrates the concepts in this assumption and shows the impact of different Poincaré curves.

Assumption 2: Given a planar hybrid automaton $\mathcal{S}_{H}$, let

$$
\begin{aligned}
\tilde{G} & =\left\{x \in G \mid \nabla g(x) \cdot f_{2}(x)<0\right\}, \\
T_{2} & =\inf _{x \in \tilde{G}} \tau_{S_{p}}^{f_{2}}(x), \text { and } \\
D & =\left\{\phi_{f_{2}}(x, h) \mid x \in \tilde{G}, 0 \leq h<T_{2}\right\} .
\end{aligned}
$$

We assume that for every point $x \in D, \tau_{S_{p}}^{f_{1}}(x)$ is finite and smaller than $\tau_{G}^{f_{1}}(x)$ (which may be infinite).

Under assumption 2, and a sufficiently small $H$ in $\mathcal{S}_{H}$, trajectories that start in $\tilde{S}_{p}$ acquire a predictable pattern, illustrated in [4, Figure 6.8]. We now define the notation and restrictions to represent these trajectories.

Given a point $x_{0} \in \tilde{S}_{p}$ and delays $h_{1}, h_{2} \geq 0$, let

$$
\begin{array}{ll}
x_{1}=P_{G}^{f_{1}}\left(x_{0}\right) & x_{2}=\phi_{f_{1}}\left(x_{1}, h_{1}\right) \\
x_{3}=P_{G}^{f_{2}}\left(x_{2}\right) & x_{4}=\phi_{f_{2}}\left(x_{3}, h_{2}\right) .
\end{array}
$$

If $h_{1}<\tau_{G}^{f_{1}}\left(x_{1}\right)$ and $h_{2}<T_{2}$, by assumption 2 the following trajectory is admitted by planar $\mathcal{S}_{\max \left(h_{1}, h_{2}\right)}$.

$$
\begin{aligned}
\mathcal{T} \mathcal{Q}\left(x_{0}, h_{1}, h_{2}\right)= & \mathcal{T} P_{G}^{f_{1}}\left(x_{0}\right) \uplus \mathcal{T} \phi_{f_{1}}\left(h_{1}, x_{1}\right) \uplus \mathcal{T} P_{G}^{f_{2}}\left(x_{2}\right) \\
& \uplus \mathcal{T} \phi_{f_{2}}\left(h_{2}, x_{3}\right) \uplus \mathcal{T} P_{S_{p}}^{f_{1}}\left(x_{4}\right) .
\end{aligned}
$$

We also introduce the notation

$$
\mathcal{Q}\left(x_{0}, h_{1}, h_{2}\right) \triangleq P_{S_{p}}^{f_{1}}\left(x_{4}\right),
$$

illustrated in Fig. 2a 


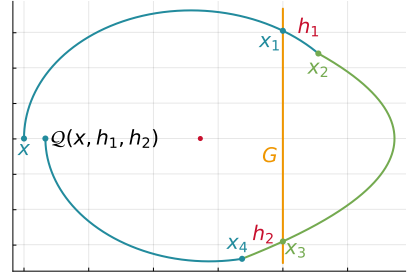

(a) Illustration of $\mathcal{Q}\left(x, h_{1}, h_{2}\right)$.

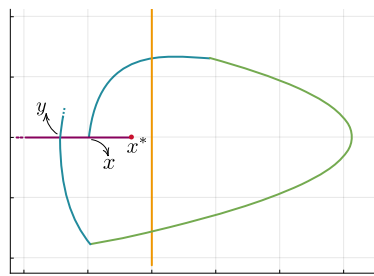

(b) Illustration of Lemma 3
Fig. 2: Illustration of the mapping $\mathcal{Q}\left(x, h_{1}, h_{2}\right)$, and Lemma 3, for the bouncing ball example (Section II). The horizontal axis refers to position, and vertical to velocity.

The following lemma is illustrated in Fig. 2b

Lemma 3: Given a delay $H<T_{2}$ (where $T_{2}$ is defined in (4)), a planar hybrid automaton $\mathcal{S}_{H}$ as given in Definition 5. with a Poincaré curve $S_{p}$ as defined in (2). Under assumptions 1 and 22 if the equilibrium $x^{*}$ is not GAS for $\mathcal{S}_{H}$, and $\mathcal{S}_{H}$ does not admit any closed orbit, then there exists two points $x, y \in \tilde{S}_{p}$ and delays $0 \leq h_{1}, h_{2} \leq H$ such that $y=\mathcal{Q}\left(x, h_{1}, h_{2}\right)$ and $\left\|y-x^{*}\right\|_{2}>\left\|x-x^{*}\right\|_{2}$.

The following lemma shows that, by adjusting the delay in $\mathcal{S}_{H}$, one can find admissible trajectories whose successive intersections with the Poincaré curve are getting closer to the equilibrium. The lemma statement is illustrated in [4, Figure $6.11]$.

Lemma 4: Given a delay $H<T_{2}$ (where $T_{2}$ is defined in (4)), a planar hybrid automaton $\mathcal{S}_{H}$ as given in Definition 5. with a Poincaré curve $S_{p}$ as defined in (2). Under assumption 1, if there exist two points $x, y \in \tilde{S}_{p}$ and delays $0 \leq h_{1}, h_{2} \leq H$ such that $y=\mathcal{Q}\left(x, h_{1}, h_{2}\right)$ and $\left\|y-x^{*}\right\|_{2}>\left\|x-x^{*}\right\|_{2}$ then there exist delays $0 \leq \overline{h_{1}}, \underline{h_{1}} \leq$ $h_{1}$ and $0 \leq \overline{h_{2}}, \underline{h_{2}} \leq h_{2}$ such that

$$
x \in\left[\mathcal{Q}\left(x, \underline{h_{1}}, \underline{h_{2}}\right), \mathcal{Q}\left(x, \overline{h_{1}}, \overline{h_{2}}\right)\right]_{S_{p}} .
$$

From the existence of an unstable trajectory, Lemma 3 combined with Lemma 4 ensures the existence of a point $x$ such that for different delays, the next intersection with the Poincaré curve is either "above" or "below" it. We now prove the continuity of the Poincaré Map to show that there are delays such that the next intersection is exactly $x$.

The continuity of Poincaré Map is classically deduced from the Implicit Function Theorem, see e.g. [22, Section 5.2, Theorem 2.1].

Lemma 5 (Continuity of Poincaré Map): Consider two continuously differentiable functions $f, g: \mathbb{R}^{2} \rightarrow \mathbb{R}$, and let $G=\{x \mid g(x)=0\}$. For any open set $A$ such that $A \cap G=\emptyset$ : if for all $x \in A, z=P_{G}^{f}(x)$ is defined, and $\nabla g(z) \cdot f(z) \neq 0$, then the Poincaré Map $P_{G}^{f}(x)$ restricted to $A$ is continuous.

Remark 1: The continuity of the Poincaré Map cannot be readily generalized to the hybrid automaton context. Given planar $\mathcal{S}_{H}$, the Poincaré Map may have discontinuities in $x$ and in the delay in the transition. Discontinuities in $x$ may happen if the trajectory is tangent to the switching surface while discontinuities in the delay in the transition may happen when the time spent in $\operatorname{Dom}(2)$ is exactly the delay in the transition. [4, Figure 6.13] illustrates these examples.

While the Poincaré Map is not continuous everywhere, under some assumptions that will be stated, our argument only relies on its continuity on regions where it is locally continuous.

In view of the statement of Lemma 5, we add the following two assumptions.

Assumption 3: We assume that for every point $x \in$ $\operatorname{int}(\operatorname{Dom}(2))$, the point $y=P_{G}^{f_{2}}(x)$ is such that the surface normal $\nabla g(y)$ and $f_{2}(y)$ are not perpendicular, that is, $\nabla g(y) \cdot f_{2}(y) \neq 0$.

Assumption 4: We assume that for every point $x \in \tilde{S}_{p}$, the point $y=P_{G}^{f_{1}}(x)$ is such that the surface normal $\nabla g(y)$ and $f_{1}(y)$ are not perpendicular, that is, $\nabla g(y) \cdot f_{1}(y) \neq 0$.

Lemma 6: Consider a planar BMHA $\mathcal{S}$ as given in Definition 4 satisfying assumption 3, and a Poincaré curve $S_{p}$ satisfying assumptions 2 and 4 For any point $x_{0} \in \tilde{S}_{p}$, the Poincaré Map $\mathcal{Q}\left(x_{0}, h_{1}, h_{2}\right)$ is defined and continuous with respect to $x_{0}, h_{1} \in\left[0 ; \tau_{G}^{f_{1}}\left(P_{G}^{f_{1}}(x)\right)\left[\right.\right.$ and $h_{2} \in\left[0 ; T_{2}[\right.$ where $T_{2}$ is defined in (4).

Proposition 3: Given a delay $H<T_{2}$ (where $T_{2}$ is defined in (4)), a planar hybrid automaton $\mathcal{S}_{H}$ as given in Definition 5. with a Poincaré curve $S_{p}$ as defined in (2). Under assumptions 1 to 4 if the equilibrium $x^{*} \in \mathbb{R}^{2}$ of $\mathcal{S}_{H}$ is not GAS and $H<T_{2}$, then $\mathcal{S}_{H}$ admits a closed orbit.

The above claim allows us to solve problem 1 by solving the following problem:

$$
\hat{\sigma}(\mathcal{S})=\inf _{H \geq 0} H \text { s.t. } \mathcal{S}_{H} \text { admits a closed orbit. }
$$

Theorem 1: Given a planar BMHA $\mathcal{S}$ as given in Definition 4 satisfying assumption 3 and a Poincaré curve $S_{p}$ as defined in (2) satisfying assumptions 1, 2 and 4, and $\sigma(\mathcal{S})<T_{2}$ (defined in $(4)$ ), the identity $\sigma(\mathcal{S})=\hat{\sigma}(\mathcal{S})$ holds.

Proof: The inequality $\sigma(\mathcal{S}) \leq \hat{\sigma}(\mathcal{S})$ follows from Proposition 2 and we show $\sigma(\mathcal{S}) \geq \hat{\sigma}(\mathcal{S})$ by contradiction. If $\sigma(\mathcal{S})<\hat{\sigma}(\mathcal{S})$ then there exists a delay $H$ such that $\sigma(\mathcal{S})<H<\hat{\sigma}(\mathcal{S})$. Since $\sigma(\mathcal{S})<H$, the equilibrium $x^{*}$ is not GAS for $\mathcal{S}_{H}$. Therefore, by Proposition 3, $\mathcal{S}_{H}$ admits a closed orbit. This is in contradiction with $H<\hat{\sigma}(\mathcal{S})$.

\section{Results}

This section shows how Theorem 1 can be applied to compute the MSD $\sigma(\mathcal{S})$ in problem 1. Then, it illustrates the solution procedure to the bouncing ball example, introduced in Section II

As discussed in Section IV, we can restrict our attention to orbits of the form $\mathcal{T} \mathcal{Q}\left(x, h_{1}, h_{2}\right)$ where $x \in \tilde{S}_{p}$. That is, we have

$$
\begin{aligned}
\hat{\sigma}(\mathcal{S})=\inf _{x \in \tilde{S}_{p}, h_{1}, h_{2}}\left\{\max \left(h_{1}, h_{2}\right) \mid x \in \tilde{S}_{p}\right. \\
\\
\left.h_{1}, h_{2} \geq 0, \mathcal{Q}\left(x, h_{1}, h_{2}\right)=x\right\} .
\end{aligned}
$$


This constrained 3-dimensional nonlinear optimization problem can be reduced to the following unconstrained 2dimensional nonlinear optimization problem:

$$
\hat{\sigma}(\mathcal{S})=\inf _{\left(x, h_{1}\right)} \max \left(h_{1}, h_{2}^{\star}\left(x, h_{1}\right)\right)
$$

where $h_{2}^{\star}\left(x, h_{1}\right)=\min \left\{h_{2} \mid \mathcal{Q}\left(x, h_{1}, h_{2}\right)=x\right\}$. This reduction is possible because, given fixed values of $x$ and $h_{1}$, the value of $h_{2}^{\star}\left(x, h_{1}\right)$ is straightforward to compute. Indeed, let $y \in G$ be such that $P_{S_{p}}^{f_{1}}(y)=x$, we have

$$
h_{2}^{\star}\left(x, h_{1}\right)=\tau_{\mathcal{T}}^{f_{2}}\left(x_{2}\right)-\tau_{G}^{f_{2}}\left(x_{2}\right)
$$

where $\mathcal{T}=\mathcal{T} P_{S_{p}}^{f_{1}}(y)$ and $x_{2}$ is defined in (5). For a given accuracy, $h_{2}^{\star}\left(x, h_{1}\right)$ can be computed using classical simulation methods for nonlinear systems (see e.g. [23]) as follows. For a given point $x \in \tilde{S}_{p}$, we precompute the trajectory $\mathcal{T}$ with a time steps determined by the required accuracy. Then we compute the point $\tau_{\mathcal{T}}^{f_{2}}\left(x_{2}\right)$ by first simulating the trajectory with a coarse time step. Let $t, z$ be the last element of the sampled trajectory before the intersection with $\mathcal{T}$. We have $\tau_{\mathcal{T}}^{f_{2}}\left(x_{2}\right)=t+\tau_{\mathcal{T}}^{f_{2}}(z)$ and $\tau_{\mathcal{T}}^{f_{2}}(z)$ is smaller than the time step used to simulate the trajectory starting at $x_{2}$. We can therefore estimate $\tau_{\mathcal{T}}^{f_{2}}(z)$ with a refined time step. This procedure can be applied recursively.

In the example introduced in Section II] the optimal solution of the problem in (11) with accuracy $10^{-9}$ is given at $x^{\star} \approx 0.24579453, h_{1}^{\star}=0$ and $h_{2}=h_{2}^{\star}\left(x^{\star}, 0\right) \approx$ 0.0014128697 . We illustrate the objective function along the line $h_{1}=0$ in [4, Figure 6.14]. We used [24] to simulate the nonlinear system.

[4, Section 6.5.1] provides a comparison of our algorithm with a trial-and-error approach using SpaceEx.

\section{CONCLUSION}

Motivated by practical problems in the simulation of hybrid systems, we have studied how a delay in the detection of mode transitions can impact the quality of the result of the numerical simulation. It turns out that this delay may have a crucial impact on the result, as it may turn a stable hybrid system into an unstable behaviour in the numerics.

Our goal was to study this phenomenon, and we have focused here on planar systems. A natural first research question aiming at understanding the problem is to characterize the threshold between stability and instability of the simulated trajectories, when the true system is stable. Already for this simple case, it can be hard to compute the maximal allowed delay ensuring stability of the trajectories.

We have used classical techniques in the analysis of dynamical systems, such as Poincar maps and topological arguments. However, we showed that in hybrid systems, more complex phenomena can occur, which make these classical tools insufficient to solve the problem. We pushed further these techniques, which allowed us, under mild assumptions, to compute this maximal delay for planar systems. Moreover, we showed that traditional reachability analysis techniques do not scale to solve this problem satisfactorily.
We hope that this work will provide a better understanding of the problem, of high importance in numerical simulation, and will lead to the estimation of the maximal allowed delay for more complex, or higher dimensional, hybrid systems than the ones studied here.

\section{REFERENCES}

[1] R. Jungers, The Joint Spectral Radius: Theory and Applications. Springer Science \& Business Media, 2009, vol. 385.

[2] N. W. Bauer, P. J. H. Maas, and W. P. M. H. Heemels, "Stability analysis of networked control systems: A sum of squares approach," Automatica, vol. 48, no. 8, pp. 1514-1524, 2012.

[3] F. Conti and O. Khatib, "A Framework for Real-Time Multi-Contact Multi-Body Dynamic Simulation," in Robotics Research. Cham: Springer International Publishing, 2016, vol. 114, pp. 271-287. [Online]. Available: http://link.springer.com/10. 1007/978-3-319-28872-7_16

[4] C. Gomes, "Property preservation in co-simulation." [Online]. Available: https://repository.uantwerpen.be/docman/irua/57f437/163840.pdf

[5] R. M. Jungers, A. D'Innocenzo, and M. D. Di Benedetto, "Feedback stabilization of dynamical systems with switched delays," in 2012 IEEE 51st IEEE Conference on Decision and Control (CDC). Maui, HI, USA: IEEE, Dec. 2012, pp. 1325-1330.

[6] C. Gomes, P. Karalis, E. M. Navarro-López, and H. Vangheluwe, "Approximated Stability Analysis of Bi-modal Hybrid Co-simulation Scenarios," in 1st Workshop on Formal Co-Simulation of CyberPhysical Systems. Trento, Italy: Springer, Cham, 2017, pp. 345-360.

[7] F. Giannakopoulos and K. Pliete, "Planar systems of piecewise linear differential equations with a line of discontinuity," Nonlinearity, vol. 14, no. 6, pp. 1611-1632, Oct. 2001.

[8] A. Papachristodoulou and S. Prajna, "A tutorial on sum of squares techniques for systems analysis," in American Control Conference. Portland, OR, USA: IEEE, 2005, pp. 2686-2700.

[9] A. V. Proskurnikov, "Does sample-time emulation preserve exponential stability?" 2020.

[10] A. Arapostathis and M. E. Broucke, "Stability and controllability of planar, conewise linear systems," vol. 56, no. 2, pp. 150-158.

[11] K. H. Johansson, M. Egerstedt, J. Lygeros, and S. Sastry, "On the regularization of zeno hybrid automata," Systems \& control letters, vol. 38, no. 3, pp. 141-150, 1999.

[12] - "On the regularization of Zeno hybrid automata," Systems \& Control Letters, vol. 38, no. 3, pp. 141-150, Oct. 1999.

[13] R. Goebel, R. G. Sanfelice, and A. R. Teel, Hybrid Dynamical Systems: Modeling, Stability, and Robustness. Princeton University Press, 2012. [Online]. Available: http://www.jstor.org/stable/j.ctt7s02z

[14] V. D. Blondel and J. N. Tsitsiklis, "Complexity of stability and controllability of elementary hybrid systems," Automatica, vol. 35, no. 3, pp. 479-489, 1999.

[15] X. Dai, "A Gel'fand-type spectral radius formula and stability of linear constrained switching systems," Linear Algebra and its Applications, vol. 436, no. 5, pp. 1099-1113, Mar. 2012.

[16] M. Philippe, R. Essick, G. E. Dullerud, and R. M. Jungers, "Stability of discrete-time switching systems with constrained switching sequences," Automatica, vol. 72, pp. 242-250, Oct. 2016.

[17] J. C. Lagarias and Y. Wang, "The finiteness conjecture for the generalized spectral radius of a set of matrices," Linear Algebra and its Applications, vol. 214, no. 0, pp. 17-42, 1995.

[18] C. Gomes, B. Legat, R. Jungers, and H. Vangheluwe, "Minimally Constrained Stable Switched Systems and Application to Co-simulation," in IEEE Conference on Decision and Control, Miami Beach, FL, USA, 2018, pp. 5676-5681

[19] M. W. Hirsch, S. Smale, and R. L. Devaney, Differential Equations, Dynamical Systems, and an Introduction to Chaos. Academic press, 2012.

[20] J. R. Munkres, Topology, 2nd ed. Prentice Hall, 2000.

[21] D. Liberzon, Switching in Systems and Control. Springer Science \& Business Media, 2012.

[22] C. Robinson, Dynamical Systems: Stability, Symbolic Dynamics, and Chaos. CRC press, 1998.

[23] F. E. Cellier and E. Kofman, Continuous System Simulation. Springer Science \& Business Media, 2006.

[24] C. Rackauckas and Q. Nie, "Differentialequations.jl-a performant and feature-rich ecosystem for solving differential equations in julia,' Journal of Open Research Software, vol. 5, no. 1, 2017. 


\section{APPENDIX}

Proof: Lemma 1] Let $U$ be the path component of $\mathcal{X} \backslash\left(\mathcal{T}\left[t_{1}, t_{2}\right] \mid \mathcal{X} \cup\left[x_{2}, x_{1}\right]_{S_{p}}\right)$, that contains $x^{*} \in U$. By Assumption 1, there is a path in $\overline{S_{p}}$ from $x^{*}$ to $x_{2}$. This path is contained in $\bar{U}$ as it cannot intersect the interior of $\left.\mathcal{T}\left[t_{1}, t_{2}\right]\right|_{\mathcal{X}}$ by definition of $t_{2}$. Therefore, by the injectiviy assumption of Assumption 1, the set $U$ must contain all $x \in$ $S_{p}$ such that $\left\|x-x^{*}\right\|_{2} \leq\left\|x_{2}-x^{*}\right\|_{2}$.

As $\nabla s(x) \cdot f_{1}(x)>0$ for all $x \in S_{p}$ (see (2)) and $\left\|x_{2}-x^{*}\right\|_{2} \geq\left\|x_{1}-x^{*}\right\|_{2}$, there exists $\epsilon>0$ such that $\mathcal{T}\left[t_{2}, t_{2}+\epsilon\right] \mid \mathcal{X} \cap U=\emptyset$. As the trajectory at $t_{2}+\epsilon$ is not in $U$, if $\mathcal{T}\left[t_{2}, \infty\right] \mid \mathcal{X} \cap U \neq \emptyset$ then $\left(q_{3}, t_{3}, x_{3}\right) \in \mathcal{P} \frac{\mathcal{T}}{U}\left(t_{2}\right)$ is defined. The point $x_{3}$ cannot belong to $[y, x]_{S_{p}}$ as $\nabla s(x) \cdot f_{1}(x)>0$ hence $\left.x_{3} \in \mathcal{T}\left[t_{1}, t_{2}\right]\right|_{\mathcal{X}}$. For any $\left(q, t, x_{3}\right) \in \mathcal{T}\left[t_{1}, t_{2}\right]$, we must have $q \neq q_{3}$ since by [4, Lemma 1], two trajectories in the same mode cannot intersect.

Proof: |Lemma 2 Let $U$ be the path component of $\mathcal{X} \backslash\left(\mathcal{T}\left[t_{1}, t_{2}\right] \mid \mathcal{X} \cup\left[x_{2}, x_{1}\right]_{S_{p}}\right)$ containing the equilibrium $x^{*}$. If $\mathcal{T}\left[t_{2}, \infty\right] \mid \mathcal{X} \nsubseteq \bar{U}$ then given

$$
\left.\left(q^{\prime}, t^{\prime}, x^{\prime}\right) \in \mathcal{P}_{\mathcal{T}}^{\mathcal{T}}\left[t_{1}, t_{2}\right]\right|_{\mathcal{X}} \cup\left[x_{2}, x_{1}\right]_{S_{p}}\left(t_{2}\right),
$$

we know that $\left.x^{\prime} \in \mathcal{T}\left[t_{1}, t_{2}\right]\right|_{\mathcal{X}}$ since $\nabla s(x) \cdot f_{1}(x)>0$ for all $x \in S_{p}$. As $\left.\mathcal{T}\left[t_{1}, t_{2}\right]\right|_{\mathcal{X}} \subseteq \operatorname{Dom}(1), q^{\prime}=1$ which is impossible by [4, Lemma 1]. Therefore $\left.\mathcal{T}\left[t_{2}, \infty\right]\right|_{\mathcal{X}} \subseteq \bar{U} \subseteq$ $\operatorname{Dom}(1)$.

Proof: [Lemma 3] Since $\mathcal{S}_{H}$ is not GAS, it admits a trajectory $\mathcal{T}$ that does not converge to the equilibrium $x^{*}$.

The proof is divided into two parts: first we prove that trajectory $\mathcal{T}$ contains infinitely many mode transitions. Then we use that fact to prove that successive intersections satisfy the claim in the lemma. In the second part, we make use of a result that is proved later Lemma 6. Lemma 6 does not depend on Lemma 3.

We now prove by contradiction that trajectory $\mathcal{T}$ contains infinitely many mode transitions. If it contains finitely many transitions, there is a mode $q_{\infty}$ and time $t_{\infty}$ such that, for all $(q, t, x) \in \mathcal{T}$ with $t>t_{\infty}$, we have $q=q_{\infty}$. We cannot have $q_{\infty}=1$ as the equilibrium $x^{*}$ is GAS for mode 1 . Similarly, we cannot have $q_{\infty}=2$ since the equilibrium $x^{*}$ is GAS for $\mathcal{S}$. In this case, $\left(2, t^{\prime}, x^{\prime}\right) \in \mathcal{P}_{G}^{\mathcal{T}}\left(t_{\infty}\right)$ is defined. Let $\left(2, t^{\prime \prime}, x^{\prime \prime}\right) \in \mathcal{P}_{G}^{\mathcal{T}}\left(t^{\prime}\right)$. Then, by Assumption 2 there must be $t^{\prime \prime \prime}, x^{\prime \prime \prime}$ such that $\left(1, t^{\prime \prime \prime}, x^{\prime \prime \prime}\right) \in \mathcal{T}\left[t^{\prime}, t^{\prime \prime}\right]$. This concludes the proof that trajectory $\mathcal{T}$ contains infinitely many mode transitions.

Now we focus on the second part of the proof. By Assumption 2, after each transition from mode 2 to mode 1 , the trajectory $\mathcal{T}$ must intersect the Poincaré curve before it transitions from mode 1 to mode 2 . Therefore the trajectory intersects the Poincaré curve an infinite amount of times and is in mode 1 each time it intersects it. Let $\left(1, t_{1}, x_{1}\right),\left(1, t_{2}, x_{2}\right), \ldots \in \mathcal{T}$ with $t_{1}<t_{2}<\cdots$ and $x_{1}, x_{2}, \ldots \in S_{p}$ be this sequence intersections.

There are no $i \neq j$ such that $\left\|x_{i}-x^{*}\right\|_{2}=\left\|x_{j}-x^{*}\right\|_{2}$ because, by Assumption 1. $\left\|x_{i}-x^{*}\right\|_{2}=\left\|x_{j}-x^{*}\right\|_{2}$ implies that $x_{i}=x_{j}$ which contradicts the statement that no closed orbit is admitted. There are two possible cases.
- The sequence $\left(\left\|x_{i}-x^{*}\right\|_{2}\right)_{i}$ is decreasing with $i$. Since it is bounded below by 0 , it converges. Let $n=$ $\lim _{i \rightarrow \infty}\left\|x_{i}-x^{*}\right\|_{2}$. Since the trajectory is not stable, $n>0$. By Assumption 1, there exists a unique $x \in S_{p}$ such that $\left\|x-x^{*}\right\|_{2}=n$. By Lemma 6, $\mathcal{S}_{H}$ admits a closed orbit containing $x$ which contradicts the statement. Hence this case cannot be true.

- There exists a $k$ such that $\left\|x_{k+1}-x^{*}\right\|_{2}>\left\|x_{k}-x^{*}\right\|_{2}$. Let

$$
K=\left\{k \mid\left\|x_{k+1}-x^{*}\right\|_{2}>\left\|x_{k}-x^{*}\right\|_{2}\right\} .
$$

There are two possible sub-cases.

- If there exists $k \in K, t \in \mathbb{R}, x \in \mathcal{X}$ such that $(2, t, x) \in \mathcal{T}\left[t_{k}, t_{k+1}\right]$ then we are done.

- Otherwise, for all $k \in K, x_{k+1}=P_{S_{p}}^{f_{1}}\left(x_{k}\right)$. Note that, by Corollary 2, it must be the case that the trajectory between $x_{k}$ and $x_{k+1}$ is going into the domain of mode 2 . Let $j \in K$ be such that $j+1 \notin K$, and

$$
(\tilde{q}, \tilde{t}, \tilde{x}) \in \mathcal{P}_{\left.\mathcal{T}\left[t_{j}, t_{j+1}\right]\right|_{\mathcal{X}} ^{\mathcal{T}}}\left(t_{j+1}\right),
$$

as illustrated in [4, Figure 6.10]. By Lemma 1, $\tilde{q}=$ 2 . Let $\bar{t}$ be such that $(1, \bar{t}, \tilde{x}) \in \mathcal{T}\left[t_{j}, t_{j+1}\right]$. If $\tilde{x} \in$ $\operatorname{Dom}(1)$, as in $[4$, Figure 6.10], then $\mathcal{T}[\bar{t}, \tilde{t}]$ is a closed orbit which contradicts the statement. Otherwise, $\tilde{x} \in$ $\operatorname{Dom}(2)^{1}$, as shown in [4, Figure 6.10]. Let $U$ be the path component of $\left.\mathcal{X} \backslash \mathcal{T}[\bar{t}, \tilde{t}]\right|_{\mathcal{X}}$ containing the points $x \in S_{p}$ such that $\left\|x-x^{*}\right\|_{2}<\left\|x_{j+1}-x^{*}\right\|_{2}$ and $j^{\prime}=\min \left\{k \in K \mid k>j+1,\left\|x_{k+1}-x^{*}\right\|_{2}>\right.$ $\left.\left\|x_{j+1}-x^{*}\right\|_{2}\right\}$. Since $x_{j^{\prime}+1} \notin \bar{U}$, by [4, Lemma 1], $\left(q^{\prime}, t^{\prime}, x^{\prime}\right) \in \mathcal{P}_{\left.\mathcal{T}[\bar{t}, \tilde{t}]\right|_{\mathcal{X}}}^{\mathcal{T}}\left(t_{j^{\prime}}\right)$ is defined and is such that $t_{j^{\prime}} \leq \bar{t} \leq t_{j^{\prime}+1}, q^{\prime}=1$ and there exists $\left(2, t^{\prime \prime}, x^{\prime}\right) \in$ $\mathcal{T}[\bar{t}, \tilde{t}]$. The trajectory $\mathcal{T}\left[t^{\prime \prime}, t^{\prime}\right]$ is a closed orbit which contradicts the statement.

Proof: [Lemma 4] Consider a trajectory $\mathcal{T}$ of $\mathcal{S}$ starting at $y$. Let $y_{0}=y, t_{0}=0$ and

$$
\left(1, t_{k+1}, y_{k+1}\right) \in \mathcal{P}_{S_{p}}^{\mathcal{T}}\left(t_{k}\right)
$$

for $k=0,1, \ldots$ This sequence is illustrated in Fig. 3. By Corollary 2, the sequence $\left(\left\|y_{k}-x^{*}\right\|_{2}\right)_{k}$ is non-increasing in $k$. Let $j=\inf \left\{k \mid\left\|y_{k}-x^{*}\right\|_{2}<\left\|x-x^{*}\right\|_{2}\right\}$, we know that $j<\infty$ since the equilibrium $x^{*}$ is GAS for $\mathcal{S}$. In Fig. 3 , $j=3$. We show by recurrence the existence of a sequence of delays $h_{1, k} \leq h_{1}$ and $h_{2, k} \leq h_{2}$ for $k=0,1, \ldots, j$ such that $y_{k}=\mathcal{Q}\left(x, h_{1, k}, h_{2, k}\right)$, where $y_{k}$ is defined in (12). We set $h_{1,0}=h_{1}$ and $h_{2,0}=h_{2}$. For $k \geq 0$, given $h_{1, k} \leq h_{1}$ and $h_{1, k} \leq h_{2}$, let

$$
\left.\left(q^{\prime}, t^{\prime}, x^{\prime}\right) \in \mathcal{P}_{\mathcal{T}}^{\mathcal{T}}\left(x, h_{1, k}, h_{2, k}\right)\right|_{\mathcal{X}}\left(t_{k}\right),
$$

as illustrated in Fig. 3, and

$$
\left(q^{\prime \prime}, t^{\prime \prime}, x^{\prime}\right) \in \mathcal{T} \mathcal{Q}\left(x, h_{1, k}, h_{2, k}\right),
$$

\footnotetext{
${ }^{1}$ In this case, $\mathcal{T}[\bar{t}, \tilde{t}]$ is not a closed orbit since it requires a transition between mode 2 and mode 1 at $\tilde{t}$ which is not possible since $\bar{x} \in \operatorname{Dom}(2)$.
} 
where $\mathcal{T} \mathcal{Q}\left(x, h_{1, k}, h_{2, k}\right)$ is defined in (7). By Lemma 1, we only have the following two cases to consider if $q^{\prime}=2$, $q^{\prime \prime}=1$ and $x^{\prime} \in \operatorname{Dom}(2)$ then we set $h_{2, k+1}=0$ and $h_{1, k+1}=t^{\prime \prime}-\tau_{G}^{f_{1}}(x)$; otherwise, if $q^{\prime}=1, q^{\prime \prime}=2$ and $x^{\prime} \in \operatorname{Dom}(1)$ then we set $h_{1, k+1}=h_{1, k}$ and $h_{2, k+1}=$ $t^{\prime \prime}-\left(\tau_{G}^{f_{1}}(x)+h_{1, k}+\tau_{G}^{f_{2}}\left(\phi_{f_{1}}\left(P_{G}^{f_{1}}(x), h_{1, k}\right)\right)\right)$. The lemma is proved with $\overline{h_{1}}=h_{1, j-1}, \underline{h_{1}}=h_{1, j}, \overline{h_{2}}=h_{2, j-1}$ and $\underline{h_{2}}=h_{2, j}$.

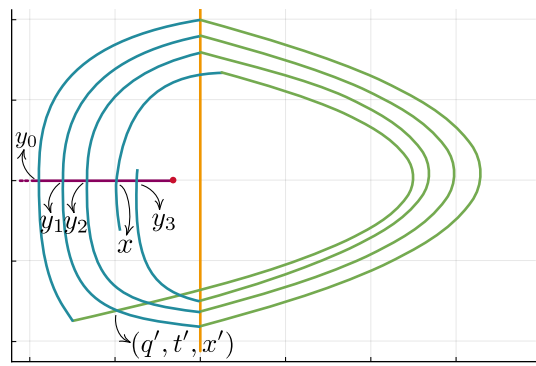

Fig. 3: Illustration of the sequence defined in (12).

Proof: [Lemma 5] Consider a point $x_{0} \in A$. Let $t_{0}=$ $\tau_{G}^{f}\left(x_{0}\right)$ and $F(x, t)=g\left(\phi_{f}(x, t)\right)$, we have $F\left(x_{0}, t_{0}\right)=0$. Furthermore

$\frac{\partial F}{\partial t}\left(x_{0}, t_{0}\right)=\nabla g\left(\phi_{f}(x, t)\right) \cdot \frac{\partial \phi_{f}(x, t)}{\partial t}=\nabla g\left(\phi_{f}(x, t)\right) \cdot f\left(\phi_{f}(x\right.$, Function Theorem [22, Section 5.2, Theorem 2.1], there exists an open set $U \subseteq \mathbb{R}^{2}$ containing $x_{0}$ and a unique con-

\footnotetext{
${ }^{2}$ Note that in both cases, we have $\mathcal{T Q}\left(x, h_{1, k+1}, h_{2, k+1}\right)=$ $\mathcal{T} \mathcal{Q}\left(x, h_{1, k}, h_{2, k}\right)\left[0, t^{\prime \prime}\right] \uplus \mathcal{T}\left[t^{\prime}, t_{k+1}\right]$.
}

tinuously differentiable function $h$ such that $F(x, h(x))=0$ for each $x \in U$. By the uniqueness of $h$, we know that $P_{G}^{f}(x)=\phi_{f}(x, h(x))$ for each $x \in U$. Since the function $h(x)$ is continuous in $x$ and the function $\phi$ is continuous in $x$ and $t$ by [4, Lemma 1], the Poincaré Map $P_{G}^{f}(x)$ is continuous in $x_{0}$.

Proof: [Lemma 6] Let $x_{1}, x_{2}, x_{3}, x_{4}$ be as defined in (5), (6) and $x_{5}$ denote $\mathcal{Q}\left(x, h_{1}, h_{2}\right)$.

By Assumption 2 and Lemma 5, $x_{5}$ depends continuously on $x_{4}$ and by [4, Lemma 1], $x_{4}$ depends continuously on $h_{2}$ hence $x_{5}$ depends continuously on $h_{2}$.

By [4, Lemma 1], $x_{4}$ depends continuously on $x_{3}$ and by Assumption 3 and Lemma 5 $x_{3}$ depends continuously on $x_{2}$ hence $x_{5}$ depends continuously on $x_{2}$.

By [4, Lemma 1], $x_{2}$ depends continuously on $h_{1}$ hence $x_{5}$ depends continuously on $h_{1}$.

By [4, Lemma 1], $x_{2}$ depends continuously on $x_{1}$ and by Assumption 4 and Lemma 5 $x_{1}$ depends continuously on $x_{0}$ hence $x_{5}$ depends continuously on $x_{0}$.

Proof: [Proposition 3] Suppose by contradiction that there exists $H$ such that $\mathcal{S}_{H}$ is not GAS but does not admit any closed orbit. Consider one such value of $H$. By Lemma 3 and Lemma 4, there a point $x \in \tilde{S}_{p}$, delays $0 \leq \overline{h_{1}}, \underline{h_{1}} \leq H$ $x$,and $0 \leq \overline{h_{2}}, \underline{h_{2}} \leq H$ such that

$$
x \in\left[\mathcal{Q}\left(x, \underline{h_{1}}, \underline{h_{2}}\right), \underline{\mathcal{Q}}\left(x, \overline{h_{1}}, \overline{h_{2}}\right)\right]_{S_{p}} .
$$

By Lemma 6, there exists $\overline{\min }\left(\overline{h_{1}}, \underline{h_{1}}\right) \leq h_{1} \leq \max \left(\overline{h_{1}}, \underline{h_{1}}\right)$ and $\min \left(\overline{h_{2}}, \underline{h_{2}}\right) \leq h_{2} \leq \overline{\max }\left(\overline{h_{2}}, \underline{h_{2}}\right)$ such that $\mathcal{Q}\left(x, h_{1}, h_{2}\right)=x$. This contradicts the fact that $\mathcal{S}_{H}$ admits no closed orbit. Hence the proof is complete. 